\title{
Expanding the use of volatile anesthetic agents beyond the operating room
}

\author{
Marcin Wąsowicz, MD, PhD • Angela Jerath, MD
}

Received: 5 June 2014/ Accepted: 14 July 2014/Published online: 30 July 2014

(C) Canadian Anesthesiologists' Society 2014

\begin{abstract}
Although volatile anesthetic agents (VAA) and conventional anesthesia workstations allowing for their delivery have been facets of modern-day operating rooms for many generations of anesthesiologists, they have also been used sporadically in the intensive care unit (ICU) with reports dating back for more than 30 years. ${ }^{1}$ The application of VAA as sedatives has been limited largely to patients suffering from severe reactive airway disease, status epilepticus, or other complex sedation scenarios. ${ }^{2}$ In spite of the success with VAA, a number of concerns have limited wider use of volatile-based sedation. Until recently, delivery of volatile-based sedation required the use of an anesthesia machine, which is difficult to accommodate within the confined space of the ICU environment ${ }^{3}$ and demands the constant presence of an anesthesiologist, neither of which is practical or feasible. In addition, concerns regarding occupational exposure to anesthetic gases have also been cited as reason for their limited uptake in the ICU.
\end{abstract}

Several of these challenges have now been overcome with the introduction of the Anaesthetic Conserving Device (AnaConDa ${ }^{\mathrm{TM}}$ ) (Sedana Medical, Sweden). The AnaConDa, an efficient lightweight and portable minivaporizer, is placed between the breathing circuit and the endotracheal tube and is universally compatible with ICU ventilators. ${ }^{3-5}$ The device is simple to use, and sedation can be easily monitored and titrated to the desired level by the nursing and respiratory therapy staff under the supervision of the attending critical care physician. Additionally, the AnaConDa is a highly efficient delivery system owing to a

M. Wąsowicz, MD, PhD (ه) · A. Jerath, MD

Department of Anesthesia and Pain Management, Toronto

General Hospital, Toronto, ON, Canada

e-mail: marcin.wasowicz@uhn.on.ca built-in carbon reflection filter that facilitates recycling more than $90 \%$ of the expired volatile agent. ${ }^{5}$

In this issue of the Journal, Ruszkai et al. describe the treatment of a patient suffering from status asthmaticus that was resistant to conventional medical therapy. ${ }^{6}$ Due to worsening hypoxemia, the patient required endotracheal intubation with subsequent mechanical ventilation. After intubation, the authors introduced treatment with sevoflurane administered via the AnaConDa. Sevoflurane, as with all VAA, provides bronchodilation which leads to decreased airway resistance and a reduction in shunt fraction. This strategy applied by Ruszkai et al. resulted in rapid relief of severe airway obstruction and simultaneously provided excellent ICU sedation which facilitated mechanical ventilation. Importantly, as previously documented, Ruszkai and coauthors showed that sedative doses of sevoflurane did not influence the patient's hemodynamic status. Relatively short treatment and ICU ventilation resulted in rapid improvement of airway pressure, pulmonary compliance, and elimination of intrinsic positive end-expiratory pressure.

The findings presented here by Ruszkai et al. confirm our previous experience with the use of inhaled VAA in the treatment of refractory asthma. ${ }^{1,6}$ But now this treatment can be applied simply and rapidly in critical care scenarios using the AnaConDa. This case report forms part of a growing body of evidence suggesting that inhaled volatile-based sedation in the ICU can potentially improve patient outcomes with better pulmonary ventilation-perfusion matching, shorter ventilation times, and good cardiovascular stability. ${ }^{3,4}$ On the other hand, the conventional sedation protocols (benzodiazepines, propofol, and opioids) are associated with high rates of oversedation, delirium, and cardiovascular depression which may potentially lead to a prolonged ICU stay and 
an increase in patient morbidity and mortality. ${ }^{7,8}$ In contrast, volatile agents can provide an improved quality of sedation with precise control of dosing (end-tidal concentration monitoring) and minimal systemic metabolism and negligible drug accumulation. This is particularly important in the context of the rapid growth in the aging and complex ICU population where hepatic and/ or renal dysfunction commonly lead to a "drug hangover" and slow drug washout times. Given their unique pharmacokinetic and pharmacodynamic properties, VAA are potentially the ideal ICU sedative. ${ }^{3,5,9}$ These characteristics of volatile anesthetics are obvious to anesthesiologists. As anesthesiologists, we have expertise in the choice and application of these medications with in-depth knowledge of their pharmacokinetic and pharmacodynamic profiles. Our expertise is based on millions of anesthetic procedures performed every year. The role of anesthesia as a specialty continues to grow beyond the operating room. Our presence has expanded throughout the hospital, including non-surgical and intensive care services. The use of volatile agents and their delivery system requires another paradigm shift in sedation practice within the ICU. Is it time to move forward and apply VAA-based sedation as part of our ICU therapy? Furthermore, is it safe and feasible for non-anesthesiologists to provide this treatment?

Several small single-centre studies have shown the advantages of VAA-based protocols for short- and long-term sedation. ${ }^{4,10,11}$ These studies investigated general medical and surgical ICU populations, including cardiac surgical and neurosurgical ICU populations. ${ }^{4,10-12}$ We have recently completed a novel North American study randomizing cardiac surgical patients to short-term intravenous propofol or inhaled volatile-based sedation, showing similar results. ${ }^{13}$ This study randomized 141 cardiac surgical patients to receive propofol-based or VA-based sedation. Patients who received VAA-based sedation achieved faster readiness for tracheal extubation and actual extubation time. Additionally, we did not notice differences between the two groups regarding hemodynamic parameters, sedation scores, or amount of postoperative analgesia. ${ }^{13}$

Prior to instituting VAA sedation more extensively into our standard ICU sedation armamentarium, these clinical outcomes must be confirmed by larger multicentre randomized trials, which are lacking for VAA-based sedation. Before embarking on these studies, there are important safety issues to address, including the risk of ambient room contamination and potential fluoride nephrotoxicity. Current Canadian regulations stipulate that the maximum levels for occupational exposure should be less than $2 \mathrm{ppm}$. In contrast, In Europe, where AnaConDa is used more frequently without additional scavenging, the limits are more liberal $(<50 \mathrm{ppm})$. We designed and tested a scavenging system connected to an ICU ventilator that minimized ambient room contamination to below required limits. ${ }^{5}$ As a result of our two studies, Health Canada gave approval for use of the AnaConDa in the ICU. The efficacy of this system was initially tested for a short-term (up to $14 \mathrm{hr}$ ) sedation trial, and testing is now being expanded to longer exposures in the Use of VAs for Long-Term Sedation in Critically Ill Patients study (VALTS) (www.clinicaltrials.gov NCT 01983800), which is currently underway. This trial is also designed to test and detect all other potential feasibility and safety issues pertaining to the use of VAA for long-term sedation, including the incidence of delirium and delivery of VAA by non-anesthesiologists. A similar trial is being conducted in Europe (EudraCT 2007-006087-30). ${ }^{14}$ Perhaps both trials will identify other limitations of VAA-based sedation, which is crucial for future multicentre randomized controlled trials.

In summary, volatile-based ICU sedation is an emerging concept for an alternative treatment within the ICU environment. The use of VAA within the ICU has moved beyond selected cases of refractory asthma and "failure to sedate" scenarios. The role of anesthesiologists in Canada has expanded and continues to grow within the field of hospital-based acute care and perioperative medicine. The uptake of VAA-based sedation within critical care units requires a cultural shift, which calls for the support and training that our discipline can provide.

\section{Étendre l'utilisation des anesthésiques volatils au-delà de la salle d'opération}

Même si les agents anesthésiques volatils (AV) et les machines d'anesthésie conventionnelles permettant leur administration ont été les facettes des salles d'opération modernes pour de nombreuses générations d'anesthésiologistes, ils ont également été utilisés de façon sporadique dans les unités de soins intensifs (USI) depuis plus de 30 ans. ${ }^{1}$ L'emploi des AV comme sédatifs a été largement limité à des patients souffrant de maladie respiratoire réactionnelle grave, de status epilepticus ou de problèmes complexes de sédation. ${ }^{2}$ En dépit du succès obtenu avec les $\mathrm{AV}$, un certain nombre de préoccupations ont limité l'utilisation étendue d'une sédation faisant appel aux AV. Jusqu'à récemment, l'administration d'une sédation reposant sur des AV nécessitait l'utilisation d'une machine d'anesthésie qu'il est difficile d'installer dans l'espace confiné de l'environnement de l'USI $^{3}$ et 
exige la présence constante d'un anesthésiologiste, limitant d'autant la praticabilité et faisabilité de leur utilisation. De plus, les préoccupations concernant l'exposition professionnelle aux gaz anesthésiques a été également citée comme motif de son adoption mitigée dans les USI.

Quelques-uns de ces problèmes ont maintenant été vaincus avec l'introduction du dispositif Anaconda ${ }^{\mathrm{TM}}$ (Sedana Medical, Suède), littéralement dispositif de conservation d'agent anesthésique (Anaesthetic Conserving Device). L'AnaConDa est un mini-vaporisateur, léger et portable, qui est placé entre le circuit respiratoire et le tube endotrachéal et a une compatibilité universelle avec les respirateurs utilisés dans les USI. ${ }^{3-5}$ Le dispositif est simple à utiliser et la sédation peut être facilement contrôlée et titrée au niveau souhaité par le personnel infirmier et les inhalothérapeutes sous la supervision de l'intensiviste. De plus, l'AnaConDa est un système d'administration très efficace grâce à un filtre intégré de réflexion au charbon qui facilite le recyclage de plus de $90 \%$ de l'agent volatil expiré. 5

Dans ce numéro du Journal, Ruszkai et coll. décrivent le traitement d'un patient présentant un status asthmaticus résistant au traitement médical conventionnel. ${ }^{6}$ Compte tenu de l'aggravation de l'hypoxémie, le patient a nécessité une intubation endotrachéale avec une assistance respiratoire mécanique subséquente. Après intubation, les auteurs ont entrepris un traitement au sevoflurane administré via le système AnaConDa. Le sevoflurane, comme tous les $\mathrm{AV}$, provoque une bronchodilatation entrainant une diminution de la résistance des voies aériennes et de la fraction de shunt. Cette stratégie utilisée par Ruszkai et coll. a permis un soulagement rapide de l'obstruction grave des voies respiratoires et a fourni simultanément une excellente sédation facilitant ainsi l'assistance respiratoire mécanique. Tel que documenté précédemment, Ruszkai et ses coauteurs ont montré que des doses sédatives de sevoflurane n'avaient pas d'influence sur le statut hémodynamique du patient. Un traitement relativement court et une assistance respiratoire à l'USI ont permis une amélioration rapide de la pression des voies aériennes, de la compliance pulmonaire et une élimination de la pression positive intrinsèque de fin d'expiration.

Les constatations présentées ici par Ruszkai et coll. confirment notre expérience antérieure de l'utilisation des $\mathrm{AV}$ inhalés dans le traitement de l'asthme réfractaire. ${ }^{1,6} \mathrm{Ce}$ traitement peut cependant être utilisé aujourd'hui dans des situations cliniques à l'USI, simplement et efficacement, grâce à l'AnaConDa. Ce rapport de cas s'intègre dans un ensemble grandissant de données probantes suggérant qu'une sédation basée sur des agents volatils inhalés à l'USI peut améliorer l'évolution du patient avec un meilleur ratio ventilation/perfusion, un raccourcissement des durées d'assistance respiratoire mécanique et une bonne stabilité cardiovasculaire. ${ }^{3,4}$ D'un autre côté, les protocoles conventionnels de sédation (benzodiazépines, propofol et opioïdes) sont associés à des taux élevés de sédation excessive, de délirium et de dépression cardiovasculaire qui peuvent conduire à une prolongation du séjour à l'USI et à une augmentation de la morbidité et mortalité des patients. ${ }^{7,8}$ En revanche, les AV peuvent procurer une meilleure qualité de sédation avec un contrôle précis de la dose administrée (monitorage de la concentration en fin d'expiration), un métabolisme systémique minime et une accumulation négligeable du médicament. Cela est particulièrement important dans un contexte de croissance rapide à l'USI d'une population vieillissante ayant des problèmes complexes, notamment des troubles fonctionnels hépatiques et/ou rénaux, entrainant fréquemment une sensation de «gueule de bois » due au médicament ainsi que des délais d'élimination prolongés. Compte tenu de leurs propriétés pharmacodynamiques et pharmacocinétiques uniques, les $\mathrm{AV}$ sont potentiellement les sédatifs idéaux à l'USI. ${ }^{3,5,9}$ Ces caractéristiques des VA sont évidentes pour les anesthésiologistes. En tant qu'anesthésiologistes, nous sommes experts dans le choix et l'utilisation de ces médicaments ayant une connaissance approfondie de leurs profils pharmacocinétiques et pharmacodynamiques. Notre expertise repose sur les millions de procédures anesthésiques pratiquées chaque année. Le rôle de l'anesthésie comme spécialité continue à croître au-delà de la salle d'opération. Notre présence s'est étendue dans tout l'hôpital, y compris dans les services de soins non chirurgicaux et dans les services de soins intensifs. L'utilisation des AV et de leurs systèmes d'administration nécessite un autre changement de paradigme dans la pratique de la sédation au sein d'une USI. Est-il temps d'aller de l'avant et d'appliquer une sédation à base d'AV dans le cadre de nos interventions effectuées à l'USI ? De plus, est-il sécuritaire et faisable pour des non-anesthésiologistes d'administrer ce traitement?

Plusieurs petites études unicentriques ont montré les avantages de protocoles basés sur les AV pour une sédation à court et à long terme. ${ }^{4,10,11}$ Ces études ont porté sur des populations de patients admis dans des USI médicales et chirurgicales, y compris des USI de chirurgie cardiaque et neurochirurgicale. ${ }^{4,10-12}$ Nous avons récemment terminé une étude nord-américaine ayant randomisé des patients suivant chirurgie cardiaque soit pour recevoir une sédation à court terme à base de propofol intraveineux ou à base d'AV. ${ }^{13}$ Cent quarante et un patients ont été inclus dans cette étude qui a permis d'observer des résultats comparables entre les groupes. Les patients ayant reçu une sédation à base d'AV étaient plus rapidement prêts à 
l'extubation trachéale et le délai véritable d'extubation était plus court. De plus, nous n'avons pas constaté de différence entre les deux groupes concernant les paramètres hémodynamiques, les scores de sédation et la quantité d'analgésie postopératoire administrée. ${ }^{13}$

Avant d'inclure la sédation par AV de façon élargie dans notre arsenal standard de sédation à l'USI, ces résultats cliniques doivent être confirmés par de plus grandes études multicentriques randomisées, lesquelles manquent encore pour la sédation par AV. Néanmoins, nous devons répondre à d'importantes questions de sécurité, telles que le risque de contamination de l'air ambiant et la néphrotoxicité potentielle des fluorures, avant d'entreprendre de telles études. Les règlements actuels au Canada stipulent que les taux maximums d'exposition professionnelle doivent être inférieurs à XX à YY ppm, dépendamment des provinces. En Europe, en revanche, où l'AnaConDa est plus souvent utilisé sans système d'élimination des gaz, les seuils sont plus élevés $(<50 \mathrm{ppm})$. Nous avons conçu et testé un système d'élimination des gaz relié à un respirateur utilisé à l'USI qui a permis de minimiser la contamination de l'air ambiant en dessous des limites requises. ${ }^{5}$ À la suite de nos deux études, Santé Canada a donné son approbation pour l'utilisation de l'AnaConDa à l'USI. L'efficacité de ce système a été initialement testée dans le cadre d'une étude sur la sédation à court terme (jusqu'à $14 \mathrm{~h}$ ) et elle est maintenant testée avec des expositions plus prolongées dans le cadre d'une autre étude actuellement en cours, l'étude VALTS (VAs for Long-Term Sedation) chez des patients admis à l'USI (www.clinicaltrials.gov, numéro NCT 01983800). Cette étude est également conçue pour tester et détecter tous les autres problèmes éventuels de faisabilité et d'innocuité liés à l'utilisation des AV pour la sédation à long terme, y compris l'incidence du délirium et l'administration d'AV par des non-anesthésiologistes. Une étude similaire est actuellement menée en Europe (EudraCT 2007-006087-30). ${ }^{14}$ Ces deux études identifieront peut-être d'autres limites à la sédation reposant sur les AV, ce qui est crucial dans la perspective d'études randomisées multicentriques futures.

En résumé, la sédation basée sur les AV à l'USI est un concept émergent proposant une autre possibilité thérapeutique au sein d'un environnement de soins intensifs. L'utilisation d'AV à l'USI va maintenant au-delà de quelques cas sélectionnés de patients ayant un asthme réfractaire ou répondant à un scénario d' « échec de la sédation ». Le rôle des anesthésiologistes au Canada s'est élargi et continue à croître dans le domaine des soins en phase aiguë hospitalier et de la médecine périopératoire. La reprise d'une sédation à base d'AV à l'USI nécessite un changement de culture de soins nécessitant un soutien et une formation que notre discipline peut fournir.
Acknowledgements The studies that the authors of this article performed to investigate the use of VA-based sedation were conducted with peer-reviewed funding obtained from the Alternative Funding Plan for Academic Health Science Centres, Merit Award-University of Toronto, and the Canadian Anesthesiologists' Society/Canadian Anesthesia Research Foundation. No funding was received from Sedana Medical.

Conflicts of interest None declared.

Conflits d'intérêts Aucun déclaré.

\section{References}

1. O'Rourke PP, Crone RK. Halothane in status asthmaticus. Crit Care Med 1982; 10: 341-3.

2. Sackey PV, Martling CR, Radell PJ. Three cases of PICU sedation with isoflurane delivered by the 'AnaConDa'. Paediatr Anaesth 2005; 15: 879-85.

3. Meiser A, Laubenthal $H$. Inhalational anaesthetics in the ICU: theory and practice of inhalational sedation in the ICU, economics, risk-benefit. Best Prac Res Clin Anaesthesiol 2005; 19: $523-38$

4. Sackey PV, Martling CR, Granath F, Radell PJ. Prolonged isoflurane sedation of intensive care unit patients with the Anesthetic Conserving Device. Crit Care Med 2004; 32: 2241-6.

5. Pickworth T, Jerath A, DeVine R, Kherani N, Wasowicz M. The scavenging of volatile anesthetic agents in the cardiovascular intensive care unit environment: a technical report. Can J Anesth 2013; 60: 38-43

6. Ruszkai Z, Bokretas GP, Bartha PT. Sevoflurane therapy for lifethreatening acute severe asthma: a case report. Can J Anesth 2014; 60: DOI: 10.1007/s12630-014-0213-y.

7. Barr J, Fraser GL, Puntillo $K$, et al. Clinical practice guidelines for the management of pain, agitation, and delirium in adult patients in the intensive care unit. Crit Care Med 2013; 41: 263-306.

8. Friedrich JO, Baker AJ. Optimizing the approach to pain, agitation, and delirium in critical care. Can J Anesth 2014; 60: 605-10.

9. Soukup J, Scharff K, Kubosch K, Pohl C, Bomplitz M, Kompardt $J$. State of the art: sedation concepts with volatile anesthetics in critically Ill patients. J Crit Care 2009; 24: 535-44.

10. Mesnil M, Capdevila X, Bringuier $S$, et al. Long-term sedation in intensive care unit: a randomized comparison between inhaled sevoflurane and intravenous propofol or midazolam. Intensive Care Med 2011; 37: 933-41.

11. Hellstrom J, Owall A, Sackey PV. Wake-up times following sedation with sevoflurane versus propofol after cardiac surgery. Scand Cardiovasc J 2012; 46: 262-8.

12. Bosel J, Purrucker JC, Nowak F, et al. Volatile isoflurane sedation in cerebrovascular intensive care patients using AnaConDa: effects on cerebral oxygenation, circulation and pressure. Intensive Care Med 2012; 38: 1955-64.

13. Wąsowicz M, van Rensburg A, Katznelson R, Jerath A, Djaiani G. Expanding an anesthesiologist role beyond an operating room. Volatile based sedation in cardiac surgical patients. Br J Anaesth 2012; 108 (suppl 2): ii94 (abstract).

14. Soukup J, Selle A, Wienke A, Steighardt J, Wagner NM, Kellner $P$. Efficiency and safety of inhalative sedation with sevoflurane in comparison to an intravenous sedation concept with propofol in intensive care patients: study protocol for a randomized controlled trial. Trials 2012; 13: 135. 